\title{
REAC-induced endogenous bioelectric currents in the treatment of venous ulcers: a three-arm randomized controlled prospective study
}

\author{
Concettina Elio ${ }^{1}$, Vania Fontani ${ }^{2,3}$, Salvatore Rinaldi ${ }^{2,3}{ }^{凶}$, Vincenzo Gasbarro $^{4}$
}

${ }^{1}$ Interuniversity Center for Phlebolymphology, Magna Graecia University of Catanzaro, Catanzaro, Italy. ${ }^{2}$ Department of Regenerative Medicine, Rinaldi Fontani Institute, Florence, Italy. ${ }^{3}$ Research Department, Rinaldi Fontani Foundation, Florence, Italy. ${ }^{4}$ Department of Morphology, Surgery, and Experimental Medicine, Section of Clinical Surgery, University of Ferrara, Ferrara, Italy.

\begin{abstract}
Introduction: Endogenous bioelectric fields (EBFs) play a fundamental role in promoting repair and regeneration processes, including in leg venous ulcers (LVUs). Unfortunately, the mechanism underlying the production of EBFs is easily altered by infectious, traumatic, and epigenetic factors. This alteration is one of the determining factors for the chronicity of LVUs. This study investigates how radioelectric asymmetric conveyer (REAC) technology treatments, specifically designed to optimize EBFs, and in particular tissue optimization-reparative (TO-RPR) treatment, can improve the results of standard dressing with and without elastic compression in LVU patients.

Methods: A total of 30 patients were enrolled (12 males and 18 females) and randomized into three groups. All patients completed the study. Group A was treated with standard dressing, elastic compression, and REAC TO-RPR treatment; Group B was treated with standard dressing and REAC TO-RPR treatment; and Group C was treated with standard dressing and elastic compression.

Results: The results show that the combination of REAC treatment and standard dressing associated with elastic compression has the greatest therapeutic efficacy in promoting the healing process for ulcers, reducing perceived pain, and improving the quality of life of the patients treated.

Conclusions: Further studies will be useful to investigate these prospective results.
\end{abstract}

Keywords: endogenous oscillators, biostimulation, venous ulcers, function recoveries

Received: 7 May 2020 | Returned for modification: 30 June 2020 | Accepted: 3 July 2020

\section{Introduction}

Over the years there has been growing interest in the literature regarding the role of bioelectric phenomena and, in particular, endogenous bioelectric fields (EBFs) in promoting repair and regeneration processes $(1-3)$ because EBFs are able to modify and control cell behavior such as migration, proliferation, cell death, differentiation, gene expression, and shape changes (3). EBFs have also been considered as potential factors for accelerated reparative and regenerative processes in lesions of various types of tissues, such as chronic leg venous ulcers (LVUs) (4-6). LVUs are chronic and recurring skin lesions that do not tend to heal spontaneously. The estimated prevalence of LVUs in the UK population is 1.5 to 3 per 1,000 , and they comprise 80 to $85 \%$ of all leg ulcers (4). Among the various factors that can cause delay in healing, the loss of correct recovering mechanisms that support the production of EBFs can be a conditioning and determining factor $(2,7)$. Similar to other lesions, in venous ulcers too the interruption of the epithelial layers that make up the epidermis generates a steady voltage and thus an injury current across themselves, also generating a lateral electric field of 40 to $200 \mathrm{mV} / \mathrm{mm}$ (7). This phenomenon would potentially favor faster wound repair through the galvanotaxis phenomenon (8). Infectious, traumatic, and epigenetic factors can negatively affect the components that favor galvanotaxis. The inhibition of galvanotaxis means that the electric charges tend to remain localized at the level of the lesion, causing blocking or slowing down of the reparative and regenerative processes $(2,7)$. To promote reparative and regenerative phenomena, various forms of electrotherapy have been used in LVUs, but a systematic review of published literature does not make it possible to establish with certainty the real effectiveness of any of the proposed treatments because each of the studies was conducted using different methods $(4,5,9)$. Among the various methods for determining an electrical stimulation process to promote the repair or regenerative processes, research has allowed the development of a technology aimed at remodeling the EBF. This radioelectric asymmetric conveyer (REAC) technology was developed to allow the restoration of correct endogenous ion flows as important regulators of cell behavior, such as migration, proliferation, cell death, differentiation, and gene expression (10-14). Specific treatment protocols with REAC technology have also demonstrated anti-inflammatory properties $(15,16)$ and reparative or regenerative efficacy (17-19). These treatments, called tissue optimization (TO) treatments, are aimed at promoting reparative (TO-RPR) and regenerative (TO-RGN) effects.

This study investigates the combination of standard dressing treatment, with and without elastic compression, which allows the REAC TO-RPR treatment to reach the greatest efficacy in reducing healing time and consequently in reducing pain and improving the quality of life (QoL) of LVU patients. 


\section{Material and methods}

\section{Ethics}

The Institutional Review Board-Independent Ethics Committee (IRB-IEC) of the Interuniversity Center for Phlebolymphology (CIFL) approved this prospective, randomized, controlled study (approval code ER.ALL.2013.22.A). This trial was registered in the Australian New Zealand Clinical Trials Registry (ANZCTR) under the number ACTRN12616000698471. The data for the trial were collected at the Interuniversity Center for Phlebolymphology, Biomedical Research Department, San Marino.

\section{Patient recruitment and assignment}

Inclusion criteria were the presence of chronic LVUs, and no signs of intolerance or allergy to constituents of the products used. Exclusion criteria were age under 18; antibiotics, antifungal, antiviral, or chemotherapy in place; pregnancy; immunosuppressive therapy ongoing or planned in the study period; surgeries scheduled in the study period; immune system diseases; life expectancy less than 6 months; systemic infections in place; and moderate or severe cognitive impairment.

A total of 30 patients were enrolled (12 males and 18 females) with an average age of $70.97 \pm 6.52$ years. All patients completed the study. Randomization into three groups was performed with a computerized procedure. Groups A, B, and C were formed as follows. Group A consisted of five males and five females, average age $70.90 \pm 8.58$, body mass index (BMI) average 28.90 \pm 3.57 ; Group B consisted of three males and seven females, average age $71.30 \pm 5.12$, BMI average $29.30 \pm 2.41$; and Group C consisted of four males and six females, average age $70.70 \pm 6.07$, BMI average $29.10 \pm 1.85$.

Group A was treated with standard dressing, elastic compres- sion, and REAC TO-RPR treatment. Group B was treated with standard dressing and REAC TO-RPR treatment. Group C was treated with standard dressing and elastic compression (Fig. 1).

\section{Sample screening}

The ulcers in the three groups of patients showed the following clinical history: Group A had lesions on average 9.90 months \pm 4.10 (three recurrent ulcers), Group B had lesions on average 10.20 months \pm 3.85 (four recurrent ulcers), and Group C had lesions on average 9.50 months \pm 3.47 (two recurrent ulcers). In all the cases analyzed, the ulcer was located on the middle third (III M) or distal third of the leg (III D); specifically, Group A: 5 III M, 5 III D; Group B: 4 III M, 6 III D; and Group C: 4 III M, 6 III D.

For clinical evaluation of the lesions, we used a grid of the most salient features for description of the ulcer (Table 1). For each of these characteristics, we assigned a score from 1 to 4 ( 1 for the most positive aspect and 4 for the worst). The final score, obtained by the sum of the partial scores, determined the initial general state of the ulcer (minimum score of 10, indicating no presence of ulcer or ulcer healed, and maximum score of 38, indicating ulcer under severe adverse clinical condition). In detail, the initial average scores for the lesions were Group A: $25.10 \pm 4.46$; Group B: $25.40 \pm 5.06$; and Group C: $25.20 \pm 4.76$.

\section{Symptom assessment}

For evaluation of pain, we used a visual analogue scale (VAS). In this scale, the absence of pain corresponded to o and the maximum pain corresponded to 10 . The average value of the symptom pain at baseline according to VAS was $7.67 \pm 0.58$. For Group A initial average VAS $=8$, for Group B initial average VAS $=7$, and for Group C initial average VAS $=8$.

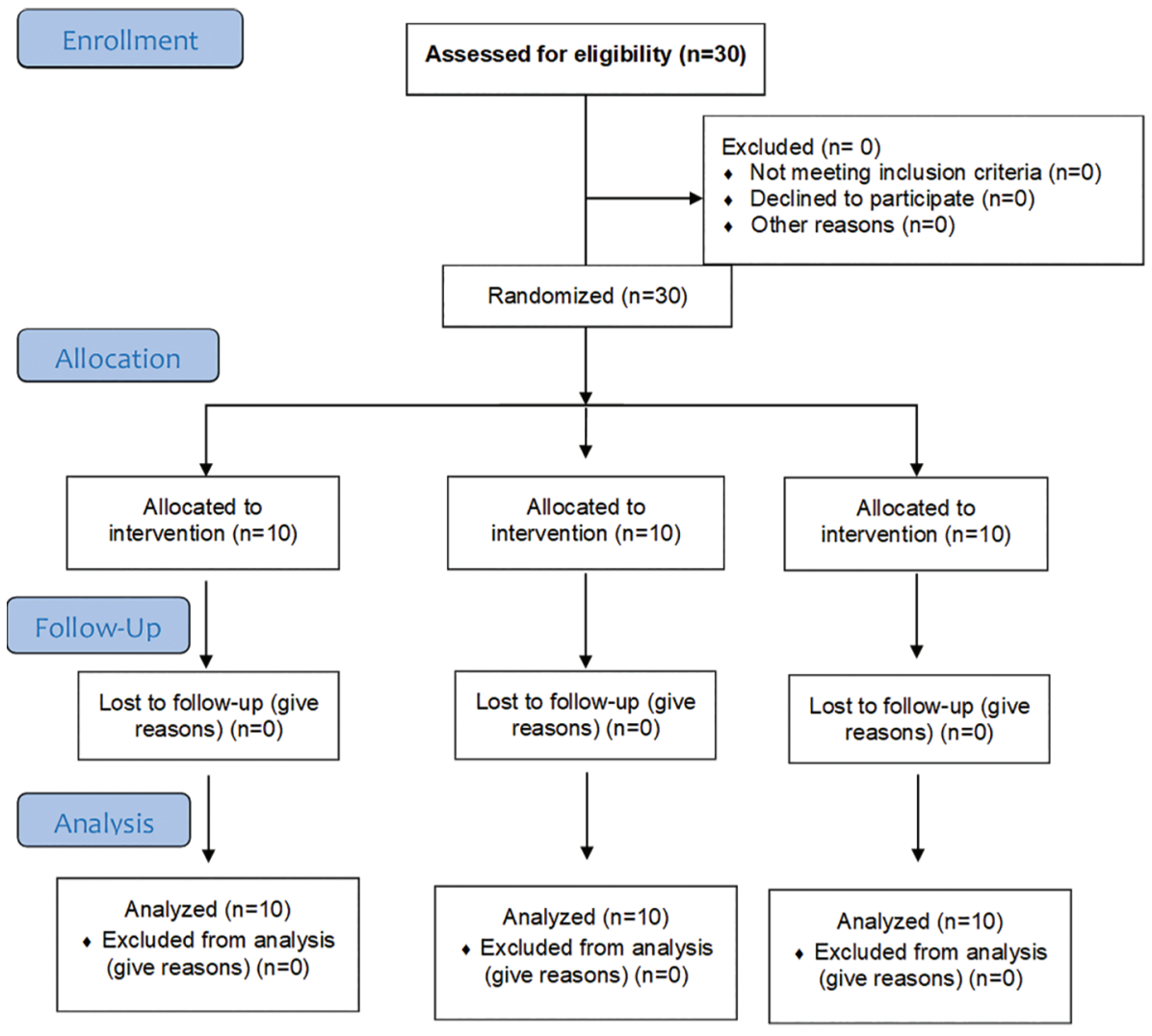

Figure 1 | CONSORT flow diagram. 
Table 1 | Clinical evaluation of the lesions.

\begin{tabular}{|c|c|c|c|c|}
\hline \multirow{2}{*}{$\frac{\text { Feature }}{\text { Redness }}$} & \multicolumn{4}{|c|}{ Clinical assessments and scores } \\
\hline & absent $=1$ & low $=2$ & moderate $=3$ & intense $=4$ \\
\hline Heat & absent $=1$ & low $=2$ & moderate $=3$ & intense $=4$ \\
\hline Functional impairment & absent $=1$ & low $=2$ & moderate $=3$ & severe $=4$ \\
\hline Exudate & absent $=1$ & low $=2$ & moderate $=3$ & abundant $=4$ \\
\hline Smell & absent $=1$ & low $=2$ & moderate $=3$ & intense $=4$ \\
\hline Granulation tissue & absent $=4$ & low $=3$ & moderate $=2$ & abundant $=1$ \\
\hline Fibrinous floor & absent $=1$ & low $=2$ & moderate $=3$ & abundant $=4$ \\
\hline Infected tissue & absent $=1$ & low $=2$ & moderate $=3$ & abundant $=4$ \\
\hline Necrotic tissue & absent $=1$ & low $=2$ & moderate $=3$ & abundant $=4$ \\
\hline Wound bed & flat $=1$ & excavated $=2$ & & \\
\hline
\end{tabular}

\section{Quality of life evaluation}

To assess a patient's QoL, the Civic 20 Q test was administered. This questionnaire analyzes aspects of the patient's life, both social and psychological. Each patient independently completed the questionnaire every 2 weeks after starting treatment. Each of the answers to the questions in the questionnaire concerning pain, daily living activities, ability to sleep overnight, and frames of mind affected by the presence of the ulcer was assigned a score (from 1 to 5, with 1 representing the minimum perceived discomfort and 5 the maximum perceived discomfort). The minimum possible score was 20 (best referable condition) and the maximum possible score was 100 (worst referable condition). The results of the analysis of the total scores obtained from each test administered at the start of the study (the average QoL scores at baseline according to Civic $20 \mathrm{Q}$ ) were Group A: $60.90 \pm 15.08$, Group B: $60.90 \pm 15.08$, and Group C: $62.00 \pm 16.22$.

\section{Body mass index}

The BMI calculation for the three groups was Group A: BMI average $28.90 \pm 3.57$, Group B: BMI average $29.30 \pm 2.41$, and Group C: BMI average $29.10 \pm 1.85$.

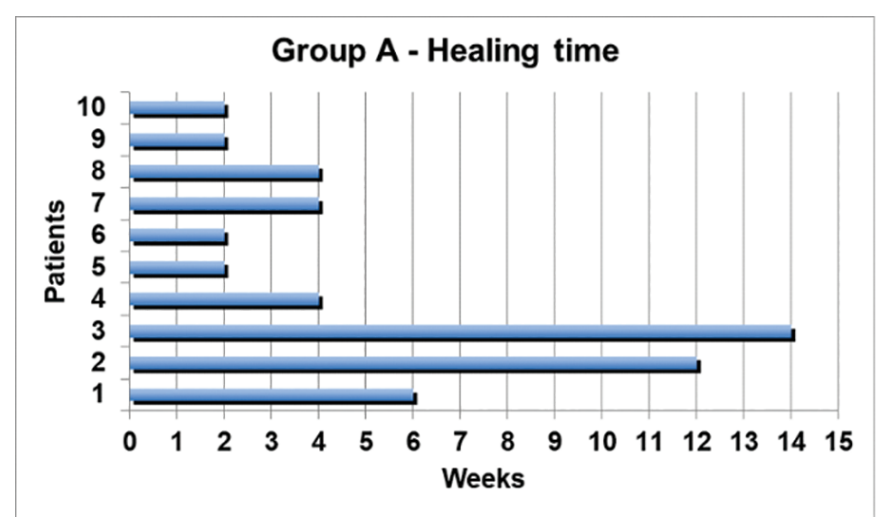

\section{Treatment procedures}

This study used the biomodulation RAEC TO-RPR treatment. REAC TO-RPR is a non-invasive and painless treatment. REAC TO-RPR treatment includes a set of treatment protocols and is administered by covering the area to be treated with a planar probe, which constitutes the asymmetric conveyer probe (ACP) connected to the REAC device.

This treatment is usually administered in multiple sessions per week, but in this study it was administered once a week, based on the replacement of dressings, for a total of 12 treatments of $15 \mathrm{~min}$ utes each. The REAC model device used in this study was BENE (ASMED, Florence, Italy).

The standard dressing for all groups consisted of cleansing with physiological solution, dressing with gauze impregnated with hyaluronic acid, and overlapping folded $10 \times 10 \mathrm{~cm}$ gauze. The bandage for Groups A and C consisted of multi-layer compression bandaging with skinsaver bandage and cohesive overlaid.

\section{Results}

In this study, the reference healing time was set to 8 weeks, based on the time taken by $80 \%$ of Group C patients to heal (Fig. 2).

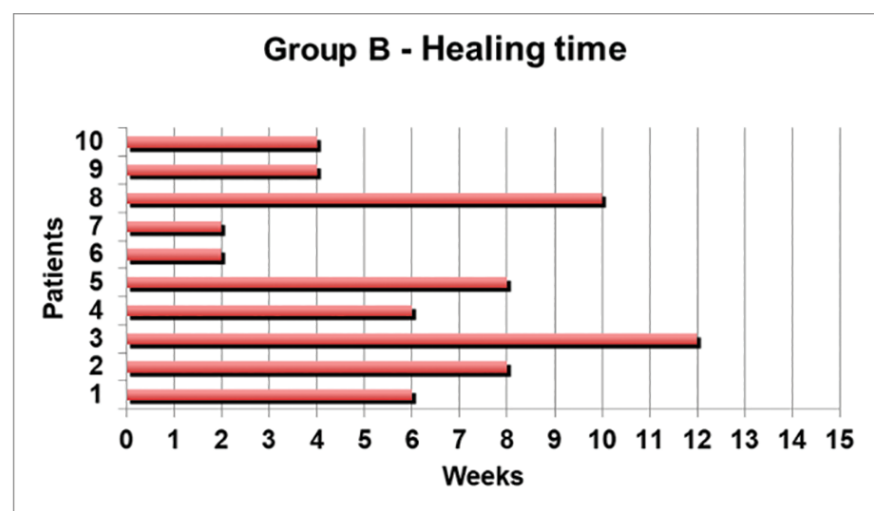

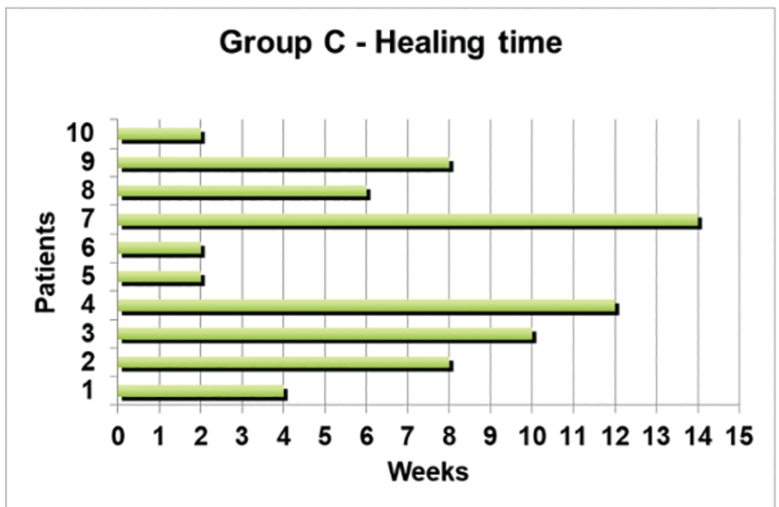

Figure 2 | Healing time of the three groups. 
With the same dressing and elastic compression, the REAC TORPR treatment for Group A resulted in halving the healing time for $70 \%$ of the patients compared to Group C, and the LVUs in Group A patients healed within the 4 th week, with an average healing time of about 20 days.

In Group B, where elastic compression was not applied, the combination of standard dressing and REAC TO-RPR slightly reduced the healing time, compared to Group C, where elastic compression was applied.

These results, albeit preliminary, show that the best combination in the treatment of LVU is standard dressing and elastic compression along with REAC TO-RPR treatment.

The symptoms of pain assessed by VAS gradually reduced in the three groups as follows. For Group A the pain disappeared after $9.40 \pm 5.40$ days, for Group B the pain disappeared after $12.00 \pm$ 5.14 days, and for Group $C$ the pain disappeared after $12.60 \pm 4.33$ days (Fig. 3a).

The three groups achieved minimum QoL scores at different timepoints. Group A achieved the result after 5.20 \pm 4.34 weeks from the start of treatment, with 64\% QoL improvement; Group B achieved the result after $6.20 \pm 3.33$ weeks from the start of treatment, with 63\% QoL improvement; and Group C achieved the result after $6.80 \pm 4.34$ weeks from the start of treatment, with $61 \%$ QoL improvement (Fig. 3b).

No side effects or adverse effects were detected in any group during the study.

\section{Discussion}

The chronic and painful nature of venous ulcers affects patients' QoL and work productivity (20). In combination with the high costs of long-term therapies, this makes venous ulcers a major health problem, especially in developed countries, where the incidence is highest (21).

The most widespread idea on the causes of chronicization of LVUs is that, once the ulcer is formed, various factors of a local or general type interfere with the tissue repair process, which seems to stop in the inflammatory phase (22). At this point, the cells undergo progressive senescence and no longer respond to growth factors and cytokines, and consequently they are no longer able to start the mitotic process (23). In general, this concept is correct, but it does not take into account alterations of the EBF components that can trigger and maintain LVUs $(7,24)$. Infectious, traumatic, and epigenetic factors can negatively affect EBFs. The inhibition of EBF signaling means that the electric charges tend to remain localized at the level of the lesion, causing the block- ing or slowing down of the reparative and regenerative processes (2). This EBF alteration triggers processes that are commonly described as etiopathogenetic factors underlying LVUs. To try to counteract this mechanism in LVUs, we used a particular treatment with REAC technology that was specifically designed to allow the restoration of EBFs as important regulators of cell behavior (10-12) and accelerate the repair process of injured tissues (17, 18). In fact, the epidermal morphology of chronic wounds differs from that of normal epidermis due to a dysregulation of signaling molecules related to a loss of cell polarity (25). In this study, we used REAC TO-RPR treatment. This treatment is usually administered in multiple sessions per week; however, it was administered only once a week in this study, based on the replacement of dressings and the scheduled periodic monitoring of patients, in order to make no changes to the treatment plan.

Evaluation of healing time showed that patients in Group A achieved faster healing than patients in Groups B and C, and that patients in Group C achieved healing more slowly than patients in Groups A and B. This suggests that the action of REAC TO-RPR treatment in combination with dressing and elastic compression contributed to expediting the wound healing process.

With respect to the symptoms of pain, patients reported a mean value of VAS of $7.67 \pm 0.58$, which was basically similar in all three groups. After treatment, all patients reported pain relief. However, the symptoms regressed differently in the three groups. Patients in Group A experienced more rapid disappearance of pain, followed by Group B, and finally Group C. This suggests that REAC TO-RPR treatment, in combination with dressing and elastic compression, is more effective in reducing the components that aggravate the pain in patients with venous ulcers. In fact, previous studies on the use of REAC technology have proven its ability to modulate neuronal circuits of pain (26).

Considering that psychosocial aspects are an important part of the treatment of venous ulcers, we wanted to investigate whether REAC TO-RPR treatment could affect a patient's QoL. For this reason, we used the Civic Q20 questionnaire. Patients in Group A experienced overall improvement in the QoL, greater than the patients in Groups B and C, and it was attained in a shorter time. Patients in Groups B and C experienced less significant improvement in QoL compared to those in Group A, and this was attained over a longer time.

The combination of REAC TO-RPR treatment with standard dressing and elastic compression achieved the best results among the three groups in this study, proving its efficacy as a therapeutic tool to improve the QoL of LVU patients. This randomized controlled prospective study showed that REAC TO-RPR treatment, in
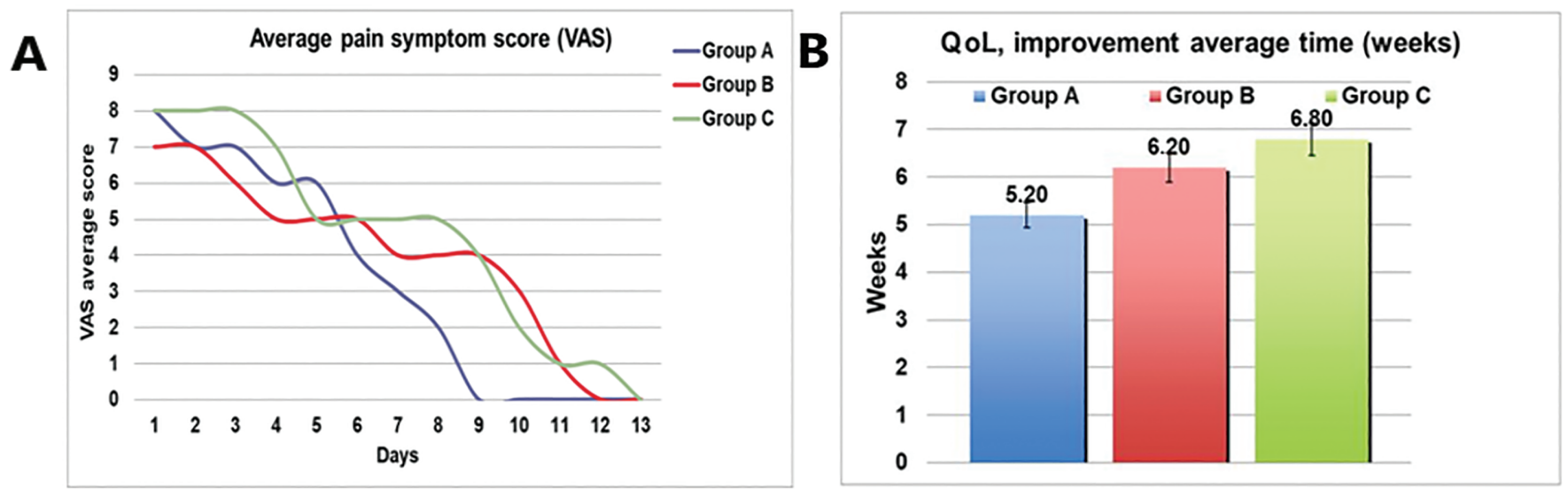

Figure 3 | Time of a) pain reduction and b) quality of life (QoL) improvement in the three groups. 
combination with dressing and elastic compression, can accelerate the healing process, reducing pain and improving the QoL of LVU patients.

\section{Conflict of interest disclosure}

There is potential conflict of interest because SR and VF are the in-

\section{References}

1. Levin M. Bioelectric mechanisms in regeneration: unique aspects and future perspectives. Semin Cell Dev Biol. 2009;20:543-56.

2. Baer ML, Colello RJ. Endogenous bioelectric fields: a putative regulator of wound repair and regeneration in the central nervous system. Neural Regen Res. 2016; 11:861-4.

3. McLaughlin KA, Levin M. Bioelectric signaling in regeneration: mechanisms of ionic controls of growth and form. Dev Biol. 2018;433:177-89.

4. Aziz Z, Cullum N. Electromagnetic therapy for treating venous leg ulcers. Cochrane Database Syst Rev. 2015:CDo02933.

5. Aziz Z, Cullum N, Flemming K. Electromagnetic therapy for treating venous leg ulcers. Cochrane Database Syst Rev. 2013:CDo02933.

6. Aziz Z, Cullum NA, Flemming K. Electromagnetic therapy for treating venous leg ulcers. Cochrane Database Syst Rev. 2011:CDo02933.

7. Nuccitelli R. A role for endogenous electric fields in wound healing. Curr Top Dev Biol. 2003;58:1-26.

8. Nuccitelli R. Physiological electric fields can influence cell motility, growth, and polarity. In: Miller KR, editor. Advances in molecular and cell biology. Burlington, MA: Elsevier; 1988. p. 213-33.

9. Ravaghi H, Flemming K, Cullum N, Olyaee Manesh A. Electromagnetic therapy for treating venous leg ulcers. Cochrane Database Syst Rev. 2006:CDo02933.

10. Maioli M, Rinaldi S, Santaniello S, Castagna A, Pigliaru G, Gualini S, et al. Radiofrequency energy loop primes cardiac, neuronal, and skeletal muscle differentiation in mouse embryonic stem cells: a new tool for improving tissue regeneration. Cell Transplant. 2012;21:1225-33.

11. Maioli M, Rinaldi S, Santaniello S, Castagna A, Pigliaru G, Gualini S, et al. Radio electric conveyed fields directly reprogram human dermal skin fibroblasts toward cardiac, neuronal, and skeletal muscle-like lineages. Cell Transplant. 2013;22:1227-35.

12. Maioli M, Rinaldi S, Santaniello S, Castagna A, Pigliaru G, Delitala A, et al. Radioelectric asymmetric conveyed fields and human adipose-derived stem cells obtained with a nonenzymatic method and device: a novel approach to multipotency. Cell Transplant. 2014;23:1489-1500.

13. Rinaldi S, Maioli M, Pigliaru G, Castagna A, Santaniello S, Basoli V, et al. Stem cell senescence. Effects of REAC technology on telomerase-independent and telomerase-dependent pathways. Sci Rep. 2014;4:6373.

14. Maioli M, Rinaldi S, Migheli R, Pigliaru G, Rocchitta G, Santaniello S, et al. Neurological morphofunctional differentiation induced by REAC technology in $\mathrm{PC}_{12}$. A neuro protective model for Parkinson's disease. Sci Rep. 2015;5:10439. ventors of REAC technology. The other authors report no conflicts of interest in this work.

\section{Acknowledgments}

We thank Alessandra Cappelli for her cooperation.
15. Lorenzini L, Giuliani A, Sivilia S, Baldassarro VA, Fernandez M, Lotti Margotti $M$, et al. REAC technology modifies pathological neuroinflammation and motor behaviour in an Alzheimer's disease mouse model. Sci Rep. 2016;6:35719.

16. Panaro MA, Aloisi A, Nicolardi G, Lofrumento DD, De Nuccio F, La Pesa V, et al. Radio electric asymmetric conveyer technology modulates neuroinflammation in a mouse model of neurodegeneration. Neurosci Bull. 2018;34:270-82.

17. Castagna A, Fontani V, Rinaldi S, Mannu P. Radio electric tissue optimization in the treatment of surgical wounds. Clin Cosmet Investig Dermatol. 2011;4:133-7.

18. Fontani V, Castagna A, Mannu P, Rinaldi S. Radioelectric asymmetric stimulation of tissues as treatment for post-traumatic injury symptoms. Int J Gen Med. 2011;4:627-34.

19. Sanna Passino E, Rocca S, Caggiu S, Columbano N, Castagna A, Fontani V, et al. REAC regenerative treatment efficacy in experimental chondral lesions: a pilot study on ovine animal model. Clin Interv Aging. 2017;12:1471-9.

20. Vishwanath V. Quality of life: venous leg ulcers. Indian Dermatol Online J. 2014; 5:397-9.

21. Criqui MH, Jamosmos M, Fronek A, Denenberg JO, Langer RD, Bergan J, et al. Chronic venous disease in an ethnically diverse population: the San Diego Population Study. Am J Epidemiol. 2003;158:448-56.

22. Ligi D, Mosti G, Croce L, Raffetto JD, Mannello F. Chronic venous disease-part I: inflammatory biomarkers in wound healing. Biochim Biophys Acta. 2016;1862: 1964-74.

23. Stanley A, Osler T. Senescence and the healing rates of venous ulcers. J Vasc Surg. 2001;33:1206-11.

24. Hunckler J, de Mel A. A current affair: electrotherapy in wound healing. J Multidiscip Healthc. 2017;10:179-94.

25. Stojadinovic O, Pastar I, Vukelic S, Mahoney MG, Brennan D, Krzyzanowska A, et al. Deregulation of keratinocyte differentiation and activation: a hallmark of venous ulcers. J Cell Mol Med. 2008;12:2675-90.

26. Zippo AG, Rinaldi S, Pellegata G, Caramenti GC, Valente M, Fontani V, et al. Electrophysiological effects of non-invasive Radio Electric Asymmetric Conveyor (REAC) on thalamocortical neural activities and perturbed experimental conditions. Sci Rep. 2015;5:18200. 